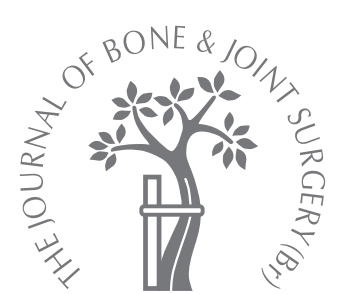

P. D. Mitchell,

N. S. Chew,

I. Goutos,

J. C. Healy,

J. C. Lee,

S. Evans,

A. Hulme

From Chelsea and

Westminster

Hospital, London, England

\footnotetext{
- P. D. Mitchell, FRCS(Trauma \& Orth), MSc, MD, Honorary Lecturer

N. S. Chew, MRCP, Radiology Specialist Registrar

I. Goutos, MRCS(Eng)

Orthopaedic Senior House

Officer

J. C. Healy, MRCP, FRCR

Consultant Radiologist

I. J. C. Lee, MRCS(Eng), FRCR,

Consultant Radiologist

- S. Evans, FRCS(Orth),

Consultant Paediatric

Orthopaedic Surgeon

A. Hulme, FRCS(Orth)

Consultant Paediatric

Orthopaedic Surgeon

Chelsea \& Westminster

Hospital, 369 Fulham Road,

London SW10 9NH, UK.

Correspondence should be sent to Mr P. D. Mitchell at The Department of Biosurgery and Surgical Technology, Imperial College London, 7E Charing Cross Hospital, Fulham Palace Road, London, W6 8RF, UK; e-mail: piers.mitchell@imperial. ac.uk
}

()2007 British Editorial Society of Bone and Joint Surgery doi:10.1302/0301-620X.89B7. $18751 \$ 2.00$

$J$ Bone Joint Surg $[\mathrm{Br}]$ 2007;89-B:948-52.

Received 6 October 2006;

Accepted after revision 20 April 2007

\section{The value of MRI undertaken immediately after reduction of the hip as a predictor of long-term acetabular dysplasia}

\begin{abstract}
Our aim was to determine whether abnormalities noted on MRI immediately after reduction for developmental dysplasia of the hip could predict the persistance of dysplasia and aid surgical planning. Scans of 13 hips in which acetabular dysplasia had resolved by the age of four years were compared with those of five which had required pelvic osteotomy for persisting dysplasia. The scans were analysed by two consultant musculoskeletal radiologists who were blinded to the outcome in each child.

The postreduction scans highlighted a number of anatomical abnormalities secondary to developmental dysplasia of the hip, but statistical analysis showed that none were predictive of persisting acetabular dysplasia in the older child, suggesting that the factors which determine the long-term outcome were not visible on these images.
\end{abstract}

Closed or open reduction in developmental dysplasia of the hip (DDH) is undertaken to facilitate normal development. ${ }^{1}$ However, some of those who undergo reduction still fail to develop a satisfactory acetabulum. Children with persisting acetabular dysplasia are commonly treated surgically since dysplasia has been shown to be associated with pain and degenerative change in early adult life. ${ }^{2}$

Our aim was to identify whether or not abnormalities seen on MRI immediately after reduction of the hip were predictive of longterm dysplasia. The advantages are that there is no need for serial pelvic radiography to assess the acetabular index, parents could be given a clear prognosis and pelvic osteotomy could be planned with confidence at an early stage.

\section{Patients and Methods}

Since 1999, all children at our hospital with displacement of the hip secondary to DDH have had MRI, while in their hip spica cast on the same day as their closed or open reduction was performed, in order to confirm satisfactory reduction of the joint. ${ }^{3-5}$ We compared the MR scans of a group which had a good outcome with those in which the acetabulum failed to develop normally. Our statistician confirmed that the review fulfilled the requirements to be classed as a prospective study. Patients excluded from the study were those over two years of age at the time of reduction, those in whom technical problems such as a movement artefact precluded detailed analysis of the MR scan, those in whom loss of reduction occurred within the hip spica and those who remained dysplastic but had not been followed up until four years of age. All the patients treated in our unit who fail to show satisfactory acetabular development by the age of four years undergo osteotomy at this stage.

Between 1999 and 2005, reduction under general anaesthesia was required for 30 displaced hips in our unit. Children presenting over the age of eight months (eight hips) did not receive any other treatment such as the use of a Pavlik harness, before reduction under general anaesthesia. The hips of all children presenting before eight months of age (ten hips) had initial treatment with a Pavlik harness. If clinical examination indicated that the hip was reduced, the harness was used for one week. The reduction and stability of the hip were reassessed clinically and by ultrasound. Some of the patients in the study had hips which were irreducible and use of the harness was not continued. Others were unstable despite treatment with the harness for one or two weeks and therefore its use was abandoned.

After reduction under general anaesthesia, all the hips were managed in a hip spica cast. In those who had open reduction (three hips) treatment in a cast continued for six weeks. Those with a closed reduction (15 hips) retained the cast for approximately six months depending on the surgeon's preference. After 


\begin{tabular}{|c|c|c|c|c|c|c|c|c|}
\hline & Patient & t Side & Gender & $\begin{array}{l}\text { Trial of Pavlik } \\
\text { harness }\end{array}$ & $\begin{array}{l}\text { Age at } \\
\text { reduction } \\
\text { (mths) }\end{array}$ & $\begin{array}{l}\text { Tenotomy at } \\
\text { reduction* }\end{array}$ & $\begin{array}{l}\text { Reduction } \\
\text { type }\end{array}$ & $\begin{array}{l}\text { Time in spica } \\
\text { (mths) }\end{array}$ \\
\hline \multirow[t]{13}{*}{ Resolving dysplasia group (13 hips, 12 patients) } & 1 & $\mathrm{~L}$ & M & $\mathrm{N}$ & 18 & No & Closed & 8.0 \\
\hline & 2 & $\mathrm{~L}$ & $\mathrm{~F}$ & $\mathrm{Y}$ & 9 & Add and pso & Open & 1.5 \\
\hline & 3 & $\mathrm{~L}$ & $\mathrm{~F}$ & $\mathrm{~N}$ & 11 & Add and pso & Closed & 6.0 \\
\hline & 4 & $\mathrm{~L}$ & $\mathrm{~F}$ & Y & 6 & Add and pso & Closed & 6.0 \\
\hline & 5 & $\mathrm{~L}$ & $\mathrm{~F}$ & $\mathrm{~N}$ & 12 & Add and pso & Closed & 6.0 \\
\hline & 6 & $\mathrm{~L}$ & $\mathrm{~F}$ & $\mathrm{~N}$ & 11 & Add and pso & Closed & 6.0 \\
\hline & 7 & $\mathrm{~L}$ & $\mathrm{~F}$ & $Y$ & 12 & Add and pso & Open & 1.5 \\
\hline & 8 & $\mathrm{R}$ & $\mathrm{F}$ & $Y$ & 12 & Add and pso & Open & 1.5 \\
\hline & 9 & $\mathrm{~L}$ & $\mathrm{~F}$ & $Y$ & 3 & Add & Closed & 6.0 \\
\hline & 10 & $\mathrm{R}$ & $\mathrm{F}$ & $\mathrm{N}$ & 21 & Add and pso & Closed & 8.0 \\
\hline & 11 & $\mathrm{~L}$ & $\mathrm{M}$ & Y & 11 & Add and pso & Closed & 6.0 \\
\hline & 12 & $\mathrm{~L}$ & $\mathrm{M}$ & Y & 6 & No & Closed & 6.0 \\
\hline & 13 & $\mathrm{~L}$ & $\mathrm{~F}$ & $\mathrm{~N}$ & 15 & Add and pso & Closed & 6.0 \\
\hline \multirow[t]{5}{*}{ Persisting dysplasia (5 hips, 4 patients) } & 14 & $\mathrm{~L}$ & $\mathrm{~F}$ & $Y$ & 12 & Add and pso & Closed & 6.0 \\
\hline & 15 & $\mathrm{R}$ & $\mathrm{F}$ & $Y$ & 12 & Add and pso & Closed & 6.0 \\
\hline & 16 & $\mathrm{R}$ & $\mathrm{M}$ & $\mathrm{N}$ & 14 & No & Closed & 6.0 \\
\hline & 17 & $\mathrm{~L}$ & $\mathrm{~F}$ & $Y$ & 6 & Add & Closed & 6.0 \\
\hline & 18 & $\mathrm{R}$ & $\mathrm{F}$ & $\mathrm{N}$ & 19 & Add and pso & Closed & 6.0 \\
\hline
\end{tabular}

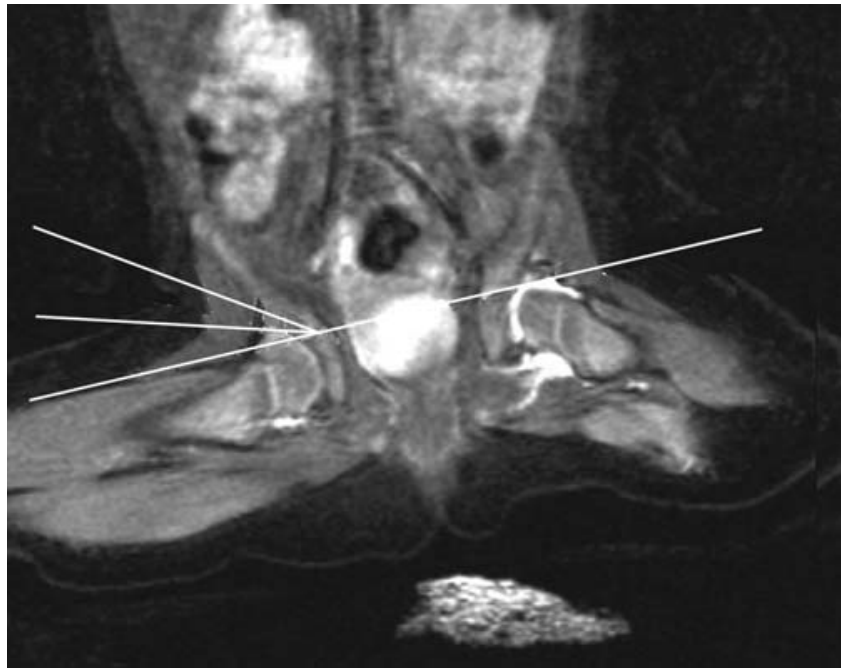

Fig. 1

Postreduction MRI showing an effusion in the left hip and the reference lines for the bony and cartilaginous acetabular index inserted by the workstation on the normal right hip.

applying the exclusion criteria outlined above, 18 hips (16 patients) were eligible for the study. They were divided into two groups for comparison. Details of age, gender, age at reduction, method of reduction, whether tenotomies were performed, and the length of time in a hip spica, are given in Table I.

Between 1999 and 2002, 13 of the 18 hips treated by reduction under general anaesthesia went on to develop a normal acetabulum. These were called the resolving dysplasia group. The last patients eligible for inclusion in this group underwent reduction in 2002 since this allowed follow-up to the age of four years.

Between 1999 and 2005, five of the 18 hips required a Salter-type pelvic osteotomy ${ }^{6}$ for persistent acetabular dysplasia. These formed the persisting dysplasia group. Initially, all had a successful closed reduction under anaesthesia. The patients in this group did not have to be followed up to the age of four years since the pelvic osteotomy was taken as the end-point of the study. As a result, patients in this group may have undergone reduction of the hip at any time between 1999 and 2005. The decision to perform a pelvic osteotomy before the age of four years was made if, after open or closed reduction and treatment in a hip spica cast, the persisting dysplasia was seen to be deteriorating on serial follow-up radiographs.

The MR scans were analysed independently by two consultant musculoskeletal radiologists (JCH, JCL) who were blinded to the outcome of each child. Between 1999 and 2003, the scans were performed on a Siemens Somaton Impact Expert 1.0 Tesla MR scanner (Siemens Medical Solutions, Erlangen, Germany). In 2003, the scanner was upgraded to a 1.5 Tesla machine (Siemens Somaton Symphony 1.5T; Siemens Medical Solutions). In all patients, the following sequences and parameters were used:

1. Coronal short-tau inversion recovery (STIR): TR 4760 ms; TE 80 ms; TI 130 ms; 3 mm slice thickness; $200 \mathrm{~mm}$ field-of-view; $256 \times 256$ matrix.

2. Axial STIR: TR $3260 \mathrm{~ms}$; TE $15 \mathrm{~ms}$; TI $130 \mathrm{~ms} ; 4 \mathrm{~mm}$ slice thickness, $200 \mathrm{~m}$ field-of-view, $256 \times 256$ matrix.

3. Axial proton density (PD): TR $4760 \mathrm{~ms}$; TE $80 \mathrm{~ms} ; 4 \mathrm{~mm}$ slice thickness; $200 \mathrm{~mm}$ field-of-view; $256 \times 256$ matrix.

Analysis was performed on the MRI workstation and note was made of a number of specific points which were thought to be predictive of long-term acetabular dysplasia. 
Table II. Acetabular labral position in the two groups after reduction

\begin{tabular}{lcl}
\hline & Resolved dysplasia & Persisting dysplasia \\
\hline Normal & 8 & 4 \\
Globular & 3 & 0 \\
Infolded & 2 & 1 \\
Total & 13 & 5 \\
\hline
\end{tabular}

Table III. Acetabular measurements in the two groups after reduction

\begin{tabular}{|c|c|c|c|c|c|c|c|}
\hline & \multicolumn{3}{|c|}{ Resolving dysplasia } & \multicolumn{3}{|c|}{ Persisting dysplasia } & \multirow{2}{*}{$\begin{array}{l}\text { Statistical p-value } \\
\text { (Mann-Whitney U } \\
\text { test) }\end{array}$} \\
\hline & Median & 25th percentile & 75th percentile & Median & 25th percentile & 75th percentile & \\
\hline Bony acetabular index & 44.0 & 41.0 & 47.0 & 38.0 & 32.5 & 43.5 & 0.70 \\
\hline Cartilage acetabular index & 21.0 & 16.5 & 25.5 & 23.0 & 21.0 & 25.0 & 0.17 \\
\hline Acetabular anteversion $\left({ }^{\circ}\right)$ & 110.0 & 104.5 & 115.5 & 112.0 & 104.5 & 119.5 & 0.85 \\
\hline $\begin{array}{l}\text { Thickness of the transverse ligament } \\
(\mathrm{mm})\end{array}$ & 2.50 & 1.35 & 3.65 & 4.30 & 2.25 & 6.35 & 0.08 \\
\hline Thickness of the pulvinar (mm) & 4.30 & 3.75 & 4.85 & 5.00 & 4.25 & 5.75 & 0.70 \\
\hline
\end{tabular}

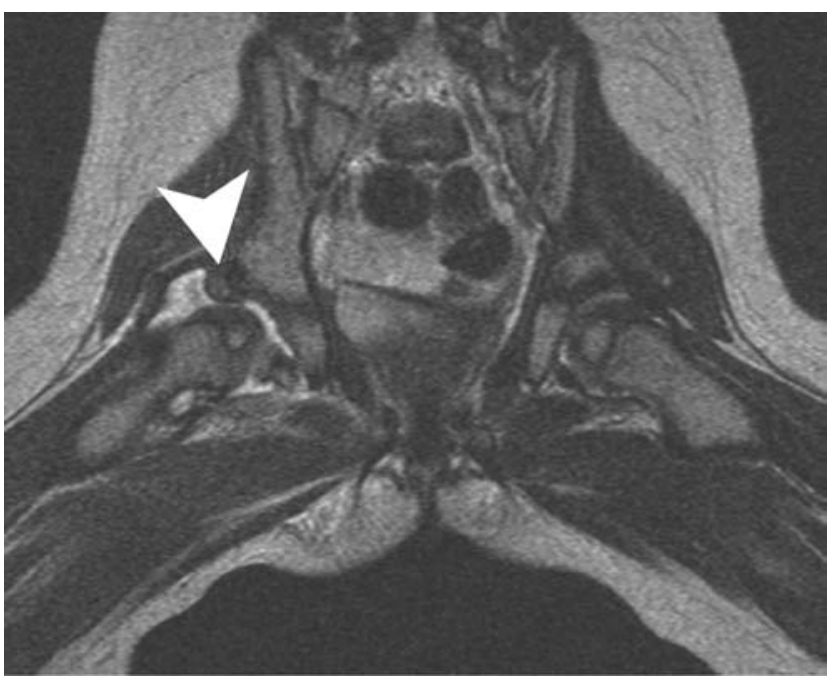

Fig. 2

MR scan showing a globular labrum and effusion in the right hip after reduction (arrowhead).

The measurements included those recommended by Duffy et $\mathrm{al}^{7}$ and comprised the shape of the cartilage anlage, the bony acetabular index, acetabular anteversion, lateral displacement resulting from panniculus, a thickened transverse ligament and whether the acetabular labrum was in a normal position or folded into the joint. The workstation was used to apply lines to each image and to measure the appropriate angles automatically (Fig. 1). The final decision as to whether the acetabular dysplasia had resolved by the age of four years was taken by one of two consultant paediatric orthopaedic surgeons (SE, AH) based on sixmonthly serial plain radiographs of the pelvis.

Statistical analysis. This was undertaken to identify whether any of the abnormalities noted on the MR scans after reduc- tion was predictive of the subsequent need for pelvic osteotomy. Comparisons of the presence or absence of a variable (i.e., labral position) were analysed by Pearson's chisquared test and of continuous data (all other measurements) by the Mann-Whitney U test. For continuous data, the median and 25 th and 75 th percentiles were given. The mean and SD were not used since it could not be determined whether the data were normally distributed about the mean.

\section{Results}

The data for the two groups are summarised in Tables II and III and examples of the pathology seen on MRI are shown in Figures 2 and 3.

Statistical analysis failed to demonstrate any difference in the two groups. They could clearly not be randomised on account of the nature of the study and therefore there was potential for them to be dissimilar and thus make comparison difficult. However, there was no statistical difference between the gender, age at reduction, method of reduction, use of tenotomies and length of time in the hip spica. Analysis also showed that none of the abnormalities noted on MRI had a statistically significant predictive value with regard to persistent acetabular dysplasia. This was because of the wide spread of the data which overlapped to a large degree between the groups. Some measurements such as the bony acetabular index (Mann-Whitney U test, $\mathrm{p}=0.70$ ), a thickened pulvinar (Mann-Whitney U test, $\mathrm{p}=0.70$ ) and acetabular anteversion (Mann-Whitney $U$ test, $p=0.85$ ) were clearly very similar between groups. Other measurements such as the thickness of the transverse ligament (Mann-Whitney U test, $\mathrm{p}=0.08$ ) and the cartilaginous acetabular index (Mann-Whitney U test, $\mathrm{p}=0.17$ ) appeared to be closer to statistical significance. A rigorous assessment was then undertaken by a senior statistician (who is not an author) to determine whether a larger sample size might have enabled these factors to be used to differentiate the 


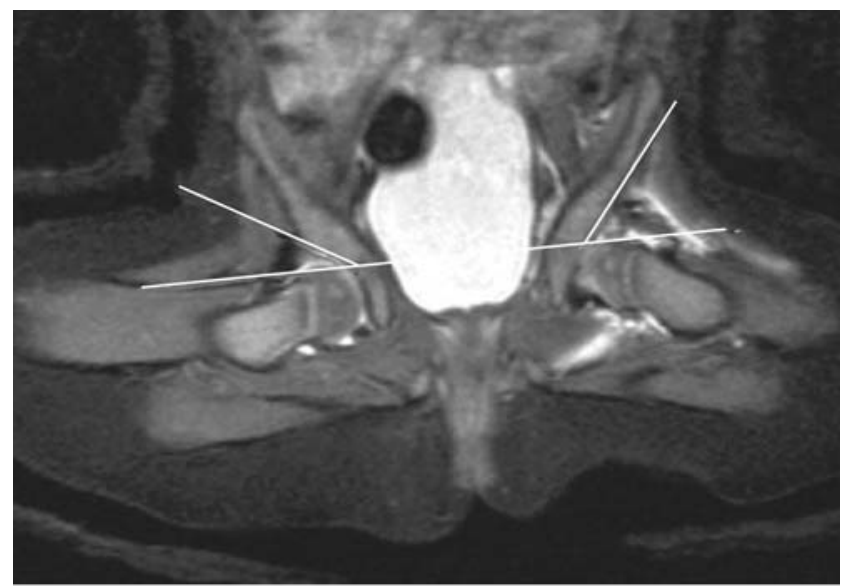

Fig. 3

MR scan showing that the bony acetabular index is abnormal (dysplastic) in the left hip but normal in the right hip.

two groups. Since power analysis is not recommended for samples of this size, a receiver operating characterisation curve analysis was performed for each variable under investigation. This highlighted the broad spread of overlapping data in both groups. The conclusion was that however large a patient group was used, there would be no clinically significant difference between the groups for any of these variables. A much larger sample size might have demonstrated a statistically significant difference between the means. However, the broad spread of the data meant that there was not a clinically useful cut-off point for any variable which would allow a clinician to determine the prognosis for dysplasia in any particular patient.

\section{Discussion}

In 2002 Duffy et al $^{7}$ studied the potential of MRI for predicting the prognosis for acetabular dysplasia after surgical management of DDH. They examined the changes in the concentricity of reduction in the first year after surgery and therefore their aims were somewhat different from those of our study. However, they commented that they were unable to predict the outcome from the initial MR scan.

Although our study had a smaller number of patients than that of Duffy et al, ${ }^{7}$ we believe that it adds to our knowledge in this field. Its principal aim was to try to identify predictive features on the initial scan which might be used to determine the prognosis for acetabular development. We followed the patients up to well-defined end-points, namely, either pelvic osteotomy for persisting acetabular dysplasia or resolution of dysplasia by the age of four years. This allowed statistical analysis to be made of possible prognostic factors between the two groups. We used computerised imaging software rather than tracing paper and pencil since we considered this to be easier to use and less prone to error.

The technique which we employed showed that a variety of anatomical changes was present in the dysplastic hip. Others have pointed out many of these in the past. ${ }^{8,9}$ MRI has also shown that dysplasia in the cartilaginous anlage tends to improve over the first year after reduction. ${ }^{7}$ It is the best method for studying the soft-tissue structures of the developing hip. We were able to avoid the need for general anaesthesia by scanning the children in their hip spica, using a quick imaging protocol.

The findings of our study were unexpected, although they confirmed the previous comments by Duffy et al. ${ }^{7}$ We anticipated that certain anatomical abnormalities in the dysplastic hip would be associated with a good or poor outcome. For example, it would be expected that the most dysplastic cartilage anlage would be most likely to lead to the greatest failure of development of the acetabulum, and that an inturned labrum would hinder normal development of the acetabulum. However, this did not appear to be the case. We were unable to identify any anatomical features which could predict whether the acetabulum would or would not remain dysplastic. This lack of statistical significance between the two groups was not a consequence of the limited sample size. Close inspection of the median and 25th and 75th percentiles in both groups showed that there was considerable overlap for the values of every analysed variable. As a result, dramatically increasing the sample size would not have identified a variable which could reliably have predicted the outcome of acetabular development.

It would appear, therefore, that it is not the anatomy of the hip which predisposes to dysplasia. It is the underlying cause of that dysplasia which produces the abnormal anatomy. Theoretical underlying causes include deficient growth factors or chemoreceptors in the cartilage anlage, dysfunctional chondrocytes or even abnormal muscle activity which may alter the biomechanics of the dysplastic hip.

Our study has highlighted a number of abnormalities in the dysplastic hip shortly after reduction. These include bony and cartilaginous dysplasia, acetabular anteversion, an infolded or globular labrum and thickening of the pulvinar and transverse ligament. However, none appear to be able to predict which hips will remain dysplastic and require pelvic osteotomy. The cause of persisting dysplasia after reduction was not identifiable on static postreduction MR scans.

We are grateful for the statistical support of Dr Elena Kulinskaya, Director of the Statistical Advice Service at Imperial College London.

No benefits in any form have been received or will be received from a commercial party related directly or indirectly to the subject of this article.

\section{References}

1. Kim HT, Kim JL, Yoo CI. Acetabular development after closed reduction of developmental dislocation of the hip. J Pediatr Orthop 2000;20:701-8. 
2. Cooperman DR, Wallensten R, Stulberg SD. Acetabular dysplasia in the adult. Clin Orthop 1983;175:79-85.

3. Giudera KJ, Einbecker ME, Berman CG, et al. Magnetic resonance imaging evaluation of congenital dislocation of the hips. Clin Orthop 1990;261:96-101.

4. McNally EG, Tasker A, Benson MK. MRI after operative reduction for developmental dysplasia of the hip. J Bone Joint Surg [Br] 1997;79-B:724-6.

5. Laor T, Roy DR, MehIman CT. Limited magnetic resonance imaging after surgica reduction of developmental dysplasia of the hip. J Pediatr Orthop 2000;20:572-4.

6. Salter RB. Innominate osteotomy in the treatment of congenital dislocation and subluxation of the hip. J Bone Joint Surg [Br] 1961;43-B:518-39.
7. Duffy CM, Taylor FN, Coleman L, Graham HK, Nattrass GR. Magnetic resonance maging evaluation of surgical management in developmental dysplasia of the hip in childhood. J Pediatr Orthop 2002;22:92-100.

8. Jaramilo D, Villegas-Medina 0, Laor T, Shapiro F, Millis BN. Gadoliniumenhanced MR imaging of pediatric patients after reduction of dysplastic hips: assessment of femoral head position, factors impeding reduction, and femoral head ischaemia. AJR 1998;170:1633-7.

9. Aoki K, Mitani S, Asaumi K, Akazawa H, Inoue H. Utility of MRI in detecting obstacles to reduction in developmental dysplasia of the hip: comparison with twodirectional arthrography and correlation with intraoperative findings. J Orthop Sci 1999;4:255-63. 